\title{
HINODE: New Space-borne Observatory for Investigating the Sun
}

\author{
By Toshifumi SHIMIZU ${ }^{1)}$ \\ ${ }^{1)}$ Institute of Space and Astronautical Science, Japan Aerospace Exploration Agency, Sagamihara, Kanagawa, Japan \\ (Received April 25th, 2008)
}

This paper gives an overview of the Hinode satellite, which was launched in September 2006 and is now observing the Sun with high spatial resolution and high performance never achieved so far. The primary aims of Hinode are to investigate magnetic activity of the Sun including its generation, energy transfer and release of the magnetic energy by simultaneously observing the solar surface (photosphere) and the corona. Some results from the Hinode observations are presented with emphasis on the supreme performance of the spacecraft and its onboard telescopes.

Key Words: Space Telescopes, Solar Physics, High Resolution

\section{Introduction}

The Sun is our closest star whose surface structures can be spatially resolved, providing a unique space plasma laboratory for investigating in detail physical mechanisms of activity and heating that occur in its magnetized atmosphere. Observations of the Sun are also able to give hints to understand the physical processes occuring on astrophysical objects, stellar evolution and galaxy formation.

Solar physics and its related research in Japan have been greatly advanced in 25 years by realizing successive series of the Sun-observing satellites; Hinotori, launched in 1981, Yohkoh ${ }^{1)}$, launched in 1991, and Hinode, launched in 2006. Yohkoh performed imaging observations of the Sun both in soft and hard X-rays and showed that the $\mathrm{X}$-ray corona is full of dynamical phenomena including flares, micro-flares, jets, and coronal restructuring. Yohkoh also provided some pieces of evidence for magnetic reconnection, which plays vital role in transiently releasing the energy of magnetic fields in the corona.

A new Sun-observing satellite Hinode (formerly Solar-B), which was successfully launched on 22 September 2006 (21:36 Universal Time), observes various types of activity and heating phenomena in the solar atmosphere with a combination of three different wavelengths; soft X-ray, extreme ultraviolet, and visible light. Thanks to its excellent high spatial and temporal resolutions, various numbers of discoveries and new knowledge on the Sun have been made from its initial observations.

\section{The Hinode spacecraft ${ }^{2)}$}

Fig. 1 is the photograph of the Hinode spacecraft taken in a clean room before installing it onto the rocket, and the external appearance of the spacecraft on orbit is illustrated in Fig. 2. The major parameters of the spacecraft are summarized in Table 1. The main structure including the onboard three telescopes has dimensions of approximately $4 \mathrm{~m} \times 1.6 \mathrm{~m} \times 1.6 \mathrm{~m}$ with two external solar panels (4.3m x $1.1 \mathrm{~m}$ each). Three telescopes (SOT, XRT, and EIS) are aligned in the $\mathrm{Z}$ axis of the spacecraft and supported by an optical bench unit (OBU), which is a cylinder made up of composite material. Most of the electronics units are located in the bus box attached to the bottom of the OBU. The OBU provides thermally and mechanically stable environment for the telescopes; the bus structure may be thermally deformed due to thermal environment change, and the micro-vibrations may be excited by attitude wheels in the bus box, but it is designed that they are not transferred to the OBU with telescopes. The solar array panels can supply about 1100W during day periods.

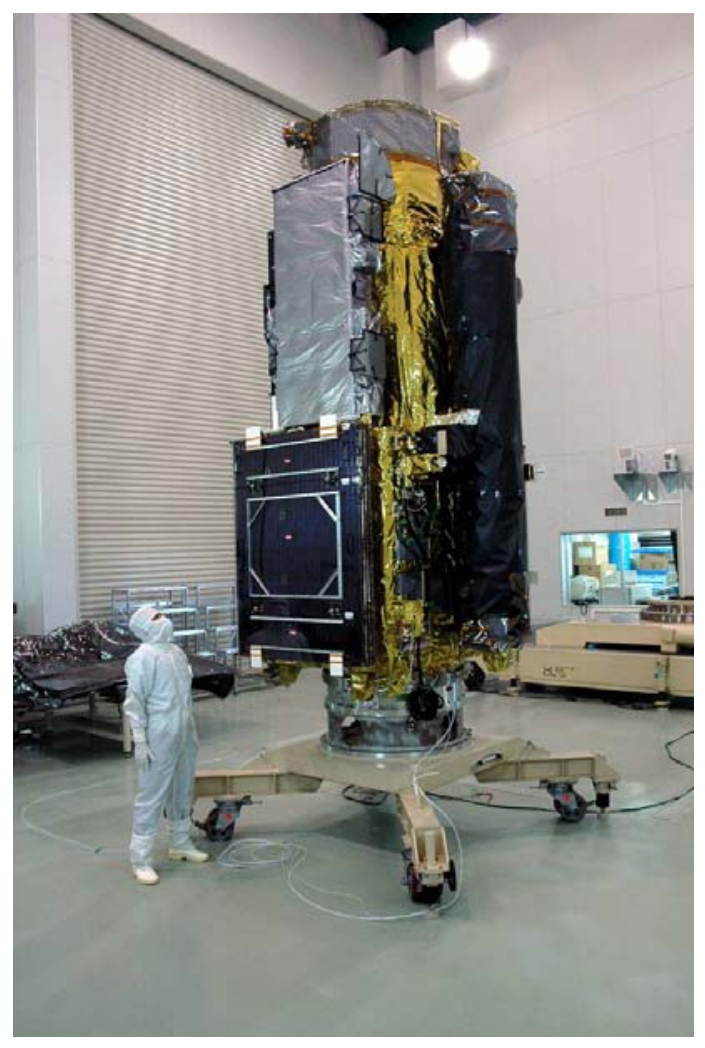

Fig. 1. The Hinode spacecraft 


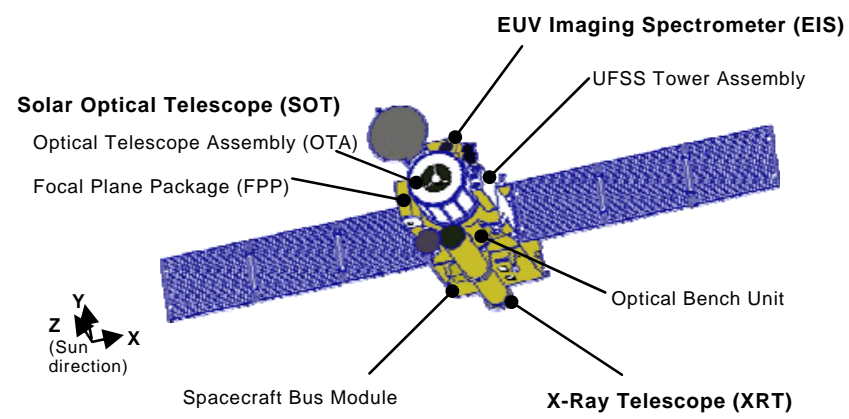

Fig. 2. External appearance of the Hinode spacecraft

Table 1. Major parameters of Hinode

\begin{tabular}{|c|c|}
\hline $\begin{array}{l}\text { Size, not including } \\
\text { solar paddles }\end{array}$ & $4000 \times 1600 \times 1600 \mathrm{~mm}$ \\
\hline Weight & 900 kg (Wet), 770 kg (Dry) \\
\hline Power & $1100 \mathrm{~W}$ \\
\hline Orbit & $\begin{array}{l}\text { Sun-synchronous polar orbit with } 680 \mathrm{~km} \\
\text { attitude and } 98.1 \mathrm{deg} \text { inclination }\end{array}$ \\
\hline $\begin{array}{l}\text { Attitude control } \\
\text { (requirement } \\
\text { parameters) }\end{array}$ & $\begin{array}{l}\text { Three-axis stabilized } \\
\text { Absolute pointing: } 20 \text { ” } \\
\text { Stability around X/Y: } 0.6 ” / 2 \mathrm{~s}, 4.5 ” / 1 \mathrm{~h} \\
\text { Stability around Z: } 200 ” / 1 \mathrm{~h} \\
\text { Pointing determination X/Y: } 0.1 \text { } \\
\text { Offset pointing: } \\
\quad \text { up to } 1178 \text { from the Sun center }\end{array}$ \\
\hline Data producing rate & $\begin{array}{l}\text { 300kbps (typical observations) } \\
\text { 2Mbps (possible peak rate) }\end{array}$ \\
\hline Data recorder & 8Gbits \\
\hline Telemetry rate & $\begin{array}{l}\text { 32K and } 256 \mathrm{Kbps} \text { (S-band) } \\
4 \mathrm{Mbps} \text { (X-band) }\end{array}$ \\
\hline Mission duration & Longer than 3 years \\
\hline
\end{tabular}

The spacecraft is stabilized by the attitude and orbit control system (AOCS) in three axes with its Z-axis pointed to the Sun. Two modes are available to direct the spacecraft to an observing target on the Sun; pointing to a fixed position and tracking a region by correcting for solar rotation. Inertial reference unit (IRU) detects changes of attitude with time with high accuracy, whereas two fine Sun sensors (UFSS) detect the absolute direction of the Sun with random noise level of 0.3 ” $(3 \sigma)$. With these attitude sensors, the AOCS succeeded to realize the requirements of stability given in Table 1 , as described later.

The orbit of the spacecraft is a Sun-synchronous polar circular orbit with attitude of $680 \mathrm{~km}$ and inclination of 98.1deg. This orbit provides a continuous view of the Sun from the spacecraft for 24 hours in about 9 months of a year. Continuous observations of the Sun are crucial in capturing dynamical behaviors of magnetic activities in various timescales from a few seconds to several days. Also, this Sun-synchronous orbit can provide a thermally stable environment, which helps to minimize the thermal deformation of the spacecraft and telescope structures.

Observations of the three telescopes are controlled by observation tables, which define the sequences of commands for controlling exposures and instrument configuration. The scientific data from the telescopes are compressed in the mission data processor (MDP) before being stored in the data recorder. Two types of compression can be performed sequentially. The first is pixel by pixel bit compression, followed by image compression (either JPEG lossy or DPCM lossless compressions), where JPEG is a lossy compression algorithm formalized by Joint Photographic Experts Group and DPCM is a lossless compression algorithm using differential pulse code modulation. The recorder memory has a total capacity of 8Gbit, which allows us to perform a continuous observation even in the periods when telemetry downlink stations are not available. The data rates from the telescopes are determined by the observation tables and compression efficiency. Typical observing run may produce $300 \mathrm{kbps}$ on average and it is possible to perform an observing run with up to $2 \mathrm{Mbps}$ peak data rate, although the data recorder memory is fulfilled with data in a short time.

\section{Scientific instruments}

Primary scientific objectives of the Hinode mission are as follows: 1) to understand the physical processes of magnetic field generation and transport including the magnetic modulation of the Sun's luminosity, 2) to investigate the processes responsible for energy transfer from the solar surface to the corona and that are responsible for the heating and structuring of the corona, and 3) to determine the physical mechanisms fundamental for the interaction between magnetic field and astrophysical plasmas, including magnetic reconnections responsible for eruptive activities, such as flares and coronal mass ejections. Moreover, it is crucial for our modern life activity to understand how the Sun affects the space environment around the earth. Hinode observations are expected to contribute to the "space weather" studies as well.

Three telescopes are onboard Hinode in order to observe the response of the corona to changes in the magnetic field at the solar surface; Solar Optical Telescope (SOT), X-Ray Telescope (XRT), and EUV Imaging Spectrometer (EIS). Major properties of the telescopes are summarized in Table 2.

The SOT is the largest solar optical telescope (with $50 \mathrm{~cm}$ clear aperture) flown in space, which consists of the Optical Telescope Assembly (OTA) and its Focal Plane Package (FPP). This telescope makes quantitative measurements of all three components of vector magnetic fields with the highest spatial resolution (0.2-0.3”) and highest accuracy $(0.1 \%)$. The continuous observations with 0.2-0.3" resolution can be achieved only from space, although a snapshot image may be acquired by the ground-based observations. The accuracy of magnetic field measurements is improved by factors of 5-10, compared with similar observations from the ground.

The response of the corona to magnetic field changes is measured by EIS and XRT with superior diagnostics 
Table 2. Major parameters of the telescopes

\begin{tabular}{|c|c|}
\hline \multicolumn{2}{|l|}{ SOT } \\
\hline Optics & Aplanatic Gregorian with $50 \mathrm{~cm}$ aperture \\
\hline Wavelength & 12 bands in $388 \mathrm{~nm}-670 \mathrm{~nm}$ \\
\hline $\begin{array}{l}\text { Sensitivity to } \\
\text { magnetic fields }\end{array}$ & $\begin{array}{l}\text { longitudinal: } 1-5 \mathrm{G} \\
\text { transverse: } 30-50 \mathrm{G}\end{array}$ \\
\hline Cadence & $\begin{array}{l}\text { 10s (filter imaging) to } \\
1 \mathrm{hr} \text { (Stokes profile mapping) }\end{array}$ \\
\hline Pixel size & $\begin{array}{l}0.053 ”(\mathrm{BFI}), 0.08 ”(\mathrm{NFI}) \\
0.16 ” \times 21.5 \mathrm{~m} \AA \text { (SP) }\end{array}$ \\
\hline Field of view & $\begin{array}{l}\text { 218” x 109” (BFI), 328” x 164” (NFI) } \\
320 ” \times 164 ”(\mathrm{SP})\end{array}$ \\
\hline \multicolumn{2}{|l|}{ XRT } \\
\hline Optics & $\begin{array}{l}\text { Modified Wolter type I grazing incidence } \\
\text { mirror ( } 35 \mathrm{~cm} \text { aperture) and co-aligned } \\
\text { optical telescope }\end{array}$ \\
\hline Wavelength & 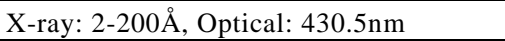 \\
\hline $\begin{array}{l}\text { Temperature } \\
\text { discrimination }\end{array}$ & $\Delta \log \mathrm{T}: 0.2$ (for isothermal plasma) \\
\hline Exposure time & $4 m s-10$ s (typical) \\
\hline Pixel size & $1.0 ”$ \\
\hline Field of view & $2048 ”$ x 2048” \\
\hline \multicolumn{2}{|l|}{ EIS } \\
\hline Optics & $\begin{array}{l}\text { Off-axis paraboloid with multilayer- } \\
\text { coated mirror and concave grating with } \\
15 \mathrm{~cm} \text { aperture }\end{array}$ \\
\hline Wavelength & $\begin{array}{l}170-210 \AA(\lambda / \Delta \lambda \sim 4000) \\
250-290 \AA(\lambda / \Delta \lambda \sim 4600)\end{array}$ \\
\hline Velocity resolution & Doppler: $3 \mathrm{~km} / \mathrm{s}$, line width: $20 \mathrm{~km} / \mathrm{s}$ \\
\hline Exposure time & ms in flares, tens of sec in active regions \\
\hline Pixel size & $1.0 ” \times 0.0223 \AA$ \\
\hline Slit/Slot width & 1”, 2”, 40”, 256” \\
\hline Field of view & $590 ” \times 512 ”$ \\
\hline
\end{tabular}

capability for plasma temperatures and motions. The XRT is a grazing incidence telescope of a Wolter I design with $30 \mathrm{~cm}$ aperture and $2.7 \mathrm{~m}$ focal length. For the highest resolution observations an on-axis focus provides an angular resolution of $\sim 1$ ”, which is at least three times better than Yohkoh. With a back-side illuminated CCD sensitive to the $\mathrm{X}$-rays longer than $50 \AA$ and careful design of X-ray analysis filters, the XRT can observe plasmas with temperatures as low as $1 \mathrm{MK}$ in the lower corona in addition to plasmas with higher temperatures between $2 \mathrm{MK}$ and $30 \mathrm{MK}$. The EIS is an imaging spectrometer designed to observe plasmas in the temperature range from $0.1 \mathrm{MK}$, the upper transition, to $3 \mathrm{MK}$, the lower corona. The EIS provides factor-of-three improvement in spatial and spectral resolution as well as by one order of magnitude higher sensitivity in detecting Doppler shift of plasma motions over a similar EUV instrument, CDS aboard the SOHO spacecraft.

\section{Technical advancement for meeting requirements}

One of important performance which is common to all the three telescopes is to continuously observe the Sun with the highest spatial resolution; 0.2-0.3” is the goal in the spatial resolution of the SOT, which is the diffraction-limited resolution at visible light range (500nm or shorter wavelength) with its $50 \mathrm{~cm}$ aperture. The spatial resolution of the XRT and EIS is 1", which is the highest spatial resolution achieved by X-ray and EUV imaging telescopes with similar designs. For achieving such performance, several technical challenges were required, which is briefly described below.

\subsection{Pointing stability requirement}

The high spatial resolution of the telescopes requires an extremely stabilized pointing of the spacecraft body, as the requirement for the attitude control is summarized in Table 1. Furthermore, the SOT requires unprecedented high pointing stability for the period of image acquisition, i.e., 0.09 " $(3 \sigma)$ for 10 sec period, which is realized by combination of three key technologies: Satellite attitude control system and an image stabilization system installed inside the SOT responsible for the frequency lower than $20 \mathrm{~Hz}$, and micro-vibration control responsible for the frequency higher than $20 \mathrm{~Hz}$.

To realize high precision in satellite attitude control, we developed attitude sensors which can provide satellite pointing information with high accuracy for controlling the satellite attitude. One of key sensors is UFSS (Ultra-Fine Sun Sensor), which was developed for Hinode by improving the performance of the former Sun sensor onboard Yohkoh. The UFSS detects the absolute direction of the Sun with random noise level of 0.3 " $(3 \sigma)$ and bias error less than 2" (p-p). The short-term requirement 0.6 " $/ 2 \mathrm{~s}$ was realized by improving the performance of both UFSS and IRU (Inertia Reference Unit) and also optimizing filter parameters used for combining signals from UFSS and IRU.

The image stabilization system installed in the SOT consists of a tip-tilt mirror located in OTA and correlation tracker in FPP. Image displacements calculated from correlation tracking of solar granules are used to control a piezo-driven tip-tilt mirror.

Micro-vibration, excited by attitude control wheels inside the satellite, may cause blur to images on the focal plane of the telescopes. Since the micro-vibration in the frequency higher than $20 \mathrm{~Hz}$ cannot be reduced by the image stabilization system and spacecraft attitude control, the structural design of the satellite and telescopes was considered to damp down micro-vibration to sufficient level. Also, the magnitude of micro-vibration from all the moving components was analytically estimated and the sources of interest for micro-vibration were identified. Some attitude control wheels were experimentally tested to know the magnitude of vibration and to reduce the micro-vibration. With the mechanical test model and flight model of the spacecraft, comprehensive measurements of the micro-vibration on the pointing stability were performed as one of major spacecraft-level pre-launch verification tests.

\subsection{Telescope co-alignment requirement}

It is also important for the three telescopes to observe 
the same target on the Sun simultaneously. The three telescopes are mounted on the OBU and their alignment relationship was measured during the pre-launch test period. The co-alignment relation is also required to be stable enough on orbit (2" or less), although the thermal environment will change repeatedly with orbital phase. The spacecraft structure was designed carefully to minimize thermal deformation due to changes of heat inputs from the environment.

\subsection{Optical space telescope for observing the Sun}

Various kinds of advanced technologies on the space optics were newly developed to achieve the diffractionlimited performance of OTA; light-weighted mirrors with extremely small deviation from the ideal optical surface, mounting mechanisms to support the mirrors without giving deformation to the mirrors, truss structures to keep the distance between primary and secondary mirrors, a collimating lens assembly which does not cause optical aberration and false polarization to the light beam, the telescope structure with the mirrors not to be vibrated by micro-vibration coming from the spacecraft, thermal design for taking care of a large amount of heat inputs from the Sun, contamination control to protect the mirrors and lens from contaminants, and so on. In order to confirm the optical performance with exposing the OTA to various thermal and mechanical environments, verification methods were also newly developed and used for pre-launch verification.

\section{Performance on orbit}

After opening protective doors in late October 2006, all the telescopes started their observations. Fig. 3 is an X-ray image of the Sun acquired by XRT. Fig. 4 and Fig. 5 are SOT images of the solar surface with 0.2 " spatial

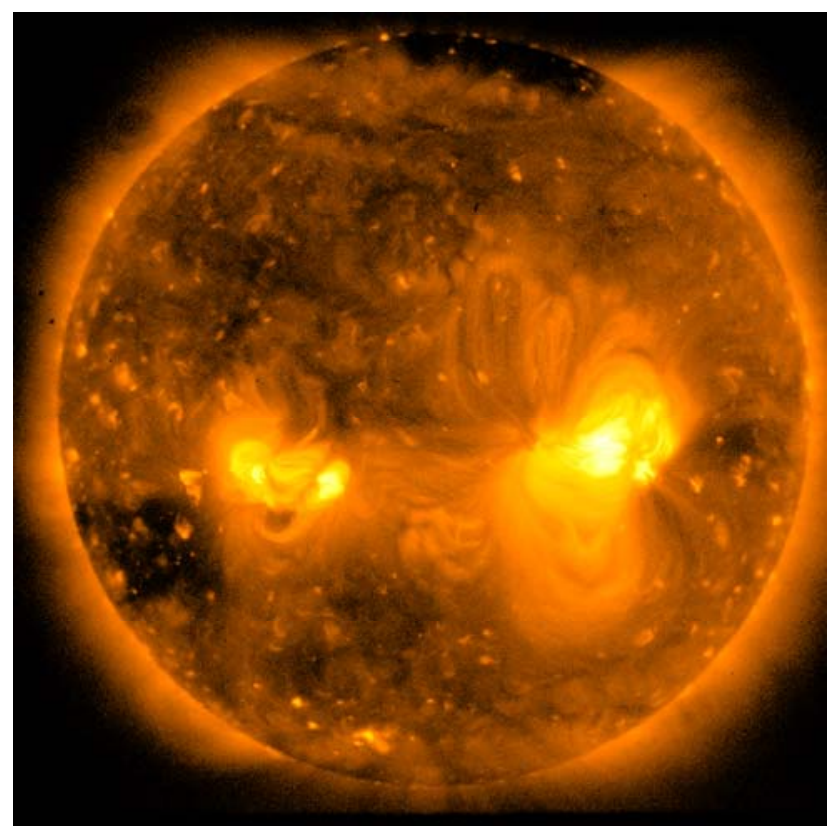

Fig. 3. An X-ray Sun image by XRT

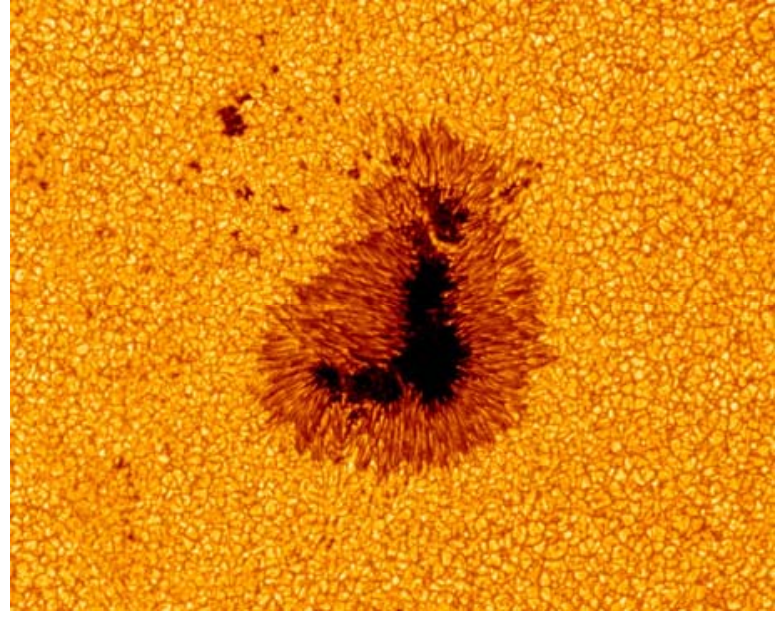

Fig. 4. A sunspot image by SOT

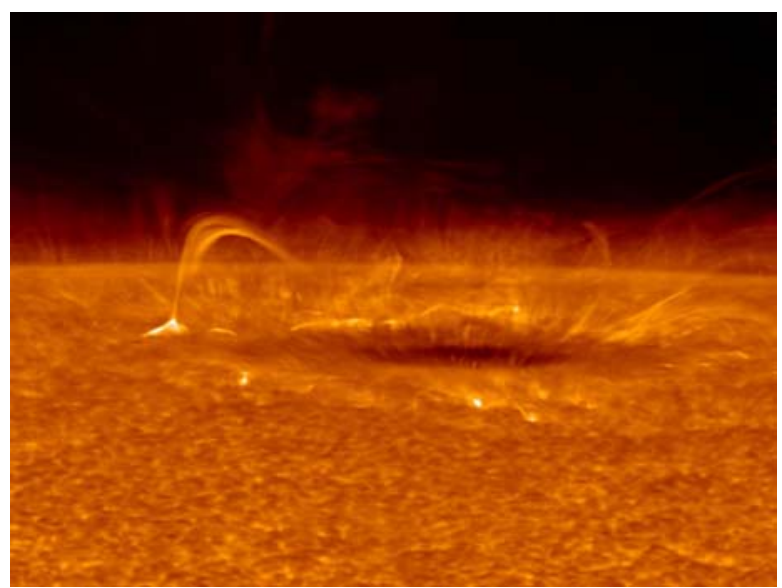

Fig. 5. Chromospheric dynamics around a sunspot located near the solar limb, acquired by SOT

resolution. These initial images have proven that they can acquire image data with supreme performances never realized so far.

Excellent pointing stability with meeting the requirement 0.09 " $(3 \sigma)$ has been making it possible for the SOT to perform its observations with 0.2 " spatial resolution and high accuracy. Fig. 6 is time profiles of the residual jitter on the focal plane for 6 hours, during which the servo is closed. The measured residual jitter is smaller than 0.03 " $(3 \sigma)$, which is much better than the requirement ${ }^{3)}$.

It has been also confirmed from the telemetry data on orbit that the spacecraft attitude is well controlled, which is easily understood from the XRT image taken with $>10$ sec exposure duration (Fig. 3).

The co-alignment among the telescopes has an excellent performance on orbit ${ }^{4)}$. Fig. 7 shows XRT and SOT pointing offsets as a function of orbital phase. Pointing jitter was removed by subtracting Sun-sensor signal, and therefore the offset of each telescope from one of the Sun sensors as the reference is derived. The XRT pointing changes as almost the same as the SOT pointing does. This clearly shows that the co-alignment among 

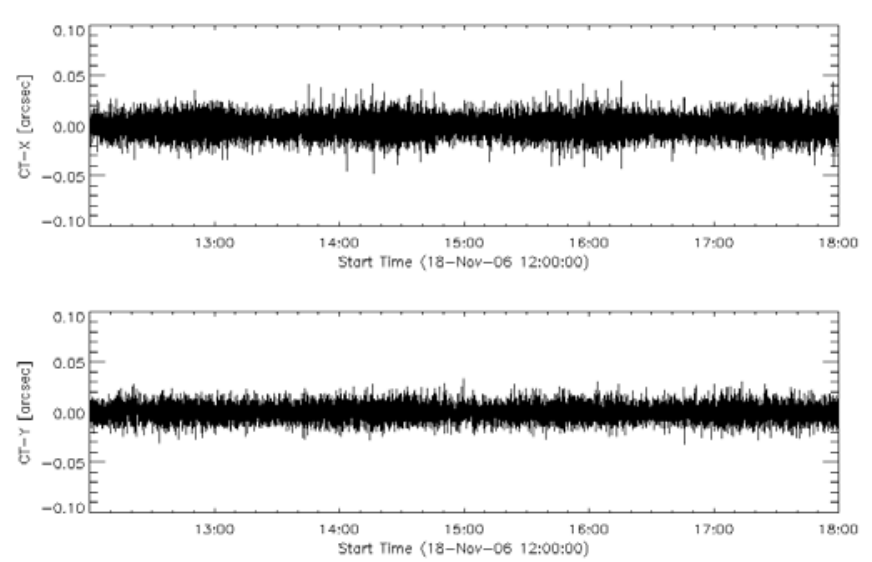

Fig. 6. Excellent SOT pointing stability is shown with time profile for each axis of the residual jitter on the focal plane for 6 hours.

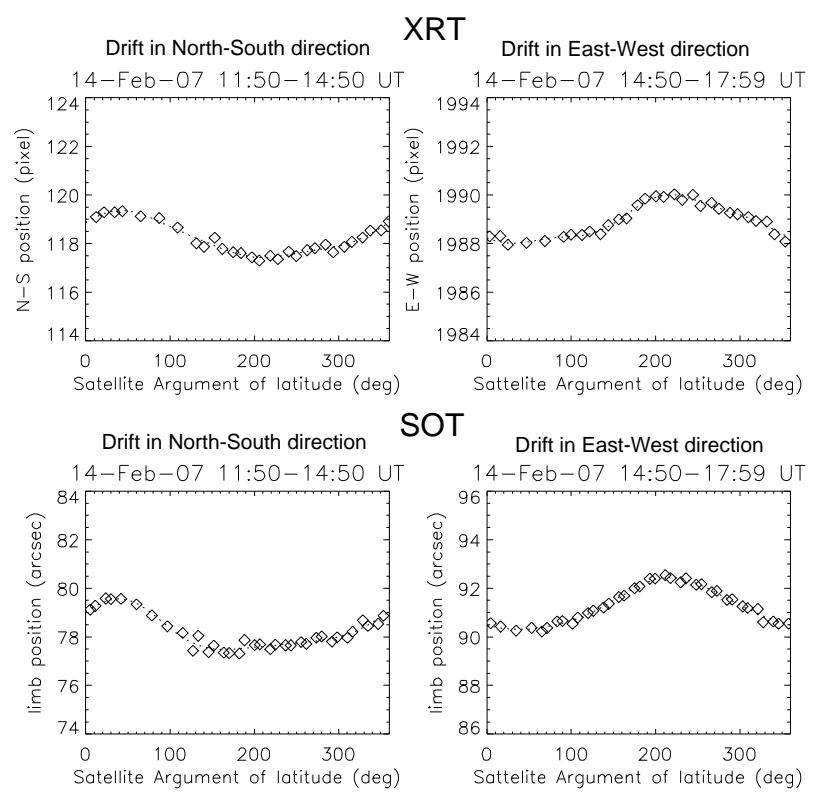

Fig. 7. XRT and SOT pointing offsets as a function of the orbital phase.

the telescopes is stable enough on orbit with the performance much better than 1 ”.

The SOT optical performance on orbit was verified with data analysis of the SOT images ${ }^{5)}$. Fig. 8 shows a closed-up image of the solar surface at $430 \mathrm{~nm}$ band (G-band) acquired with the best focus position. A bright point, marked with arrows, is one of the small features on the solar surface and it is believed to be the cross section of a tiny magnetic flux tube standing from the solar surface toward the corona. The solid line in the right panel of Fig. 8 gives intensity profile of the bright point. The dotted line is the intensity profile of a point source if observed with an ideal perfect telescope with $50 \mathrm{~cm}$ aperture.

When it is assumed that the bright point has a Gaussian profile with FWHM of 0.16 ", the ideal telescope with $50 \mathrm{~cm}$ aperture gives the intensity profile shown by dashed line. Since it is widely believed that typical bright points on the solar surface have $0.1-0.2$ ” in diameter, we can say that the optical performance of the SOT is diffraction- limited performance.
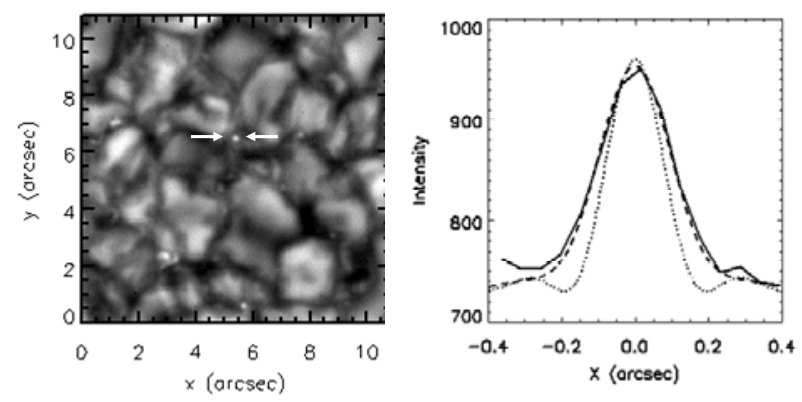

Fig. 8. High spatial resolution image at 430nm and SOT's optical performance.

\section{Scientific operations and initial results}

Scientific operations are conducted from the ISAS facility located in Sagamihara, Japan, with participation of scientists from international instrument teams. Hinode is operated as a solar observatory on orbit and its observations are scheduled with Hinode core team programs and proposal programs including coordinated observations with ground-based observatories and space missions. The data from these observations are also freely available as soon as after their acquisition. Data from Hinode is now analyzed by a lot of scientists over the world.

From observations made in initial observing phase, several numbers of discoveries have been made, such as Alfven waves in the corona ${ }^{6,7)}$, various kinds of dynamics in the chromosphere and photosphere ${ }^{8,9)}$, continuous outflowing plasma as a possible source of solar wind ${ }^{10)}$, and fine structures of magnetic field in sunspots and solar surface. They all help us to understand physical mechanisms of phenomena occurring in the solar atmosphere. Scientific results were featured as Hinode special issue in the U.S. "Science" 11), Japanese "Publications of the Astronomical Society of Japan" ${ }^{12)}$, and European “Astronomy \& Astrophysics" 13). More discoveries and new knowledge on the Sun will be expected near the future.

Hinode is a Japanese mission developed and launched by ISAS/JAXA, with NAOJ (National Astronomical Observatory of Japan) as domestic partner and NASA and STFC (UK) as international partners. It is operated by these agencies in co-operation with ESA and NSC (Norway). This presentation was made by the author in the conference on behalf of the Hinode international development team.

\section{References}

1) Ogawara, Y., Takano, T., Kato, T., Kosugi, T., Tsuntea, S., Watanabe, T., Kondo, I., and Uchida, Y.: The SOLAR-A Mission: an Overview, Solar Physics, 136 (1991), pp. 1-16.

2) Kosugi, T., Matsuzaki, K., Sakao, T., Shimizu, T., Sone, Y., 
Tachikawa, S., Hashimoto, T., Minesugi, K., Ohnishi, A., Yamada, T., Tsuneta, S., Hara, H., Ichimoto, K., Suematsu, Y., Shimojo, M., Watanabe, T., Shimada, S., Davis, J.M., Hill, L.D., Owens, J.K., Title, A.M., Culhane, J.L., Harra, L.K., Doschek, G.A., and Golub, L.: The Hinode (Solar-B) Mission: An Overview, Solar Physics, 243 (2007), pp. 3-17.

3) Shimizu, T., Nagata, S., Tsuneta, S., Tarbell, T., Edwards, C., Shine, R., Hoffmann, C., Thomas, E., Sour, S., Rehse, R., Ito, O., Kashiwagi, Y., Tabata, M., Kodeki, K., Nagase, M., Matsuzaki, K., Kobayashi, K., Ichimoto, K. and Suematsu, Y.: Image Stabilization System for Hinode (Solar-B) Solar Optical Telescope, Solar Physics, 249 (2008), pp. 221-232.

4) Shimizu, T., Katsukawa, Y., Matsuzaki, K., Ichimoto, K., Kano, R., DeLuca, E.E., Lundquist, L.L., Weber, M., Tarbell, T.D., Shine, R.A., Soma, M., Tsuneta, S., Sakao, T., and Minesugi, K.: Hinode Calibration for Precise Image Co-Alignment between SOT and XRT (2006 November-2007 April), Publications of the Astronomical Society of Japan, 59 (2007), pp. S845-S852.

5) Suematsu, Y., Tsuneta, S., Ichimoto, K., Shimizu, T., Otsubo, M., Katsukawa, Y., Nakagiri, M., Noguchi, M., Tamura, T., Kato, Y., Hara, H., Kubo, M., Mikami, I., Saito, H., Matsushita, T., Kawaguchi, N., Nakaoji, T., Nagae, K., Shimada, S., Takeyama, N., Yamamuro, T.: The Solar Optical Telescope of Solar-B (Hinode): The Optical Telescope Assembly, Solar Physics, 249 (2008), pp. 197-220.

6) De Pontieu, B., McIntosh, S.W., Carlsson, M., Hansteen, V.H., Tarbell, T.D., Schrijver, C.J., Title, A.M., Shine, R.A., Tsuneta, S., Katsukawa, Y., Ichimoto, K., Suematsu, Y., Shimizu, T., and Nagata, S.: Chromospheric Alfvenic Waves Strong Enough to Power the Solar Wind, Science, 318 (2007), pp. 1574-1577.

7) Okamoto, T.J., Tsuneta, S., Berger, T.E., Ichimoto, K., Katsukawa, Y., Lites, B.W., Nagata, S., Shibata, K., Shimizu, T., Shine, R.A., Suematsu, Y., Tarbell, T.D. and Title, A.M: Coronal Transverse Magnetohydrodynamic Waves in a Solar Prominence, Science, 318 (2007), pp. 1577-1579.
8) Shibata, K., Nakamura, T., Matsumoto, T., Otsuji, K., Okamoto, T.J., Nishizuka, N., Kawate, T., Watanabe, H., Nagata, S., UeNo, S., Kitai, R., Nozawa, S., Tsuneta, S. Suematsu, Y., Ichimoto, K., Shimizu, T., Katsukawa, Y., Tarbell, T.D., Berger, T.E., Lites, B.W., Shine, R.A. and Title, A.M.: Chromospheric Anemone Jets as Evidence of Ubiquitous Reconnection, Science, 318 (2007), pp.1591-1593.

9) Katsukawa, Y., Berger, T.E., Ichimoto, K., Lites, B.W., Nagata, S.,_Shimizu, T., Shine, R.A., Suematsu, Y., Tarbell, T.D., Title, A.M. and Tsuneta, S.: Small-Scale Jet like Features in Penumbral Chromospheres, Science, 318 (2007), pp.1594-1596.

10) Sakao, T., Kano, R., Narukage, N., Kotoku, J., Bando, T., DeLuca, E.E., Lundquist, L.L., Tsuneta, S., Harra, L.K., Katsukawa, Y., Kubo, M., Hara, H., Matsuzaki, K., Shimojo, M., Bookbinder, J.A., Golub, L., Korreck, K.E., Su, Y., Shibasaki, K., Shimizu, T., and Nakatani, I.: Continuous Plasma Outflows from the Edge of a Solar Active Region as a Possible Source of Solar Wind, Science, 318 (2007), pp.1585-1587.

11) Special section "Hinode" of Science, 318 (2007), pp.1571-1599.

12) Special issue "Initial Results from Hinode," Publications of the Astronomical Society of Japan, 59 (2007), pp. S571-S869.

13) Special feature on "Science with Hinode," Astronomy \& Astrophysics, 481 (2008), pp. L1-L72. 